\title{
Selecting Features with SVM
}

\author{
Jacek Rzeniewicz and Julian Szymański \\ Department of Computer Systems Architecture, \\ Gdańsk University of Technology, Poland \\ jrzeniewicz@gmail.com, julian.szymanski@eti.pg.gda.pl
}

\begin{abstract}
A common problem with feature selection is to establish how many features should be retained at least so that important information is not lost. We describe a method for choosing this number that makes use of Support Vector Machines. The method is based on controlling an angle by which the decision hyperplane is tilt due to feature selection.

Experiments were performed on three text datasets generated from a Wikipedia dump. Amount of retained information was estimated by classification accuracy. Even though the method is parametric, we show that, as opposed to other methods, once its parameter is chosen it can be applied to a number of similar problems (e.g. one value can be used for various datasets originating from Wikipedia). For a constant value of the parameter, dimensionality was reduced by from $78 \%$ to $90 \%$, depending on the data set. Relative accuracy drop due to feature removal was less than $0.5 \%$ in those experiments.
\end{abstract}

Keywords: feature selection, SVM, documents categorization.

\section{Introduction}

Commonly known feature selection methods for automatic text processing, like mutual information and information gain [1] [2] or SVM-based approaches [3], define ranking algorithms that allow to order features from most to least informative. However, those methods do not specify how many features must be retained for a particular set of data so that classification accuracy is preserved. This number can be determined by cross validation, yet such a solution is computationally very expensive unless the data set is small. Often either final dimensionality is fixed a priori, only features ranked above certain threshold are retained, or the number of selected features depends on vectors' density [4.

In this paper we would like to propose a different approach to deciding how many features to retain. The method is based on Support Vector Machines (SVM) 5] 6], requiring a hyperplane trained on all features. Similarly to other widely used schemes, it has a single parameter, yet we argue that it is much easier to find its correct value than in case of feature weight threshold or the final dimensionallity itself. It is worth noting that our approach does not enforce any particular ranking method. On the other hand, since it requires a hyperplane separating the classes, it would be a natural choice to take that hyperplane into account when ranking features.

J. Ruiz-Shulcloper and G. Sanniti di Baja (Eds.): CIARP 2013, Part I, LNCS 8258, pp. 319-325, 2013.

(C) Springer-Verlag Berlin Heidelberg 2013 
Much of our current work is related to processing text data obtained from Wikipedia dumps. We work on a large-scale, distributed multilabel text classifier 7 based on the LIBLINEAR library 8 and one-vs-all approach that allows to categorize a textual data into Wikipedia categories. In this context we managed to successfully apply the feature selection method described here.

In the next section we will describe the feature selection method in detail. In section 3 results of experiments conducted using presented method will be shown, and we will conclude in section 4 .

\section{Feature Selection}

One of the method's prerequisites is an SVM-trained hyperplane separating between some two classes. A normal to this hyperplane will be denoted by $\boldsymbol{w}$, while $w_{i}$ will mean an $i$ th component of $\boldsymbol{w}$ referring to feature $i$. Also a feature ranking is required in form of a vector $\boldsymbol{r}$ where $r_{i}$ denotes $i$ th feature's rank. Number of retained features is set to minimum $k$ satisfying inequality 1 .

$$
\cos \gamma^{(k)} \geq 1-\alpha
$$

where $\alpha \in[0,1]$ is the method's parameter defining the accepted level of distortion between original hyperplane and the one obtained after feature selection, and $\gamma$ is a following angle:

$$
\gamma^{(k)}=\angle\left(\boldsymbol{w}, \boldsymbol{w}^{(k)}\right)
$$

where $\boldsymbol{w}^{(\boldsymbol{k})}$ is a vector defined as below:

$$
\boldsymbol{w}^{(\boldsymbol{k})}{ }_{i}= \begin{cases}w_{i} & \text { if } r_{i} \text { is among } k \text { top components of } \boldsymbol{r} \\ 0 & \text { otherwise }\end{cases}
$$

A simple way to obtain $\boldsymbol{r}$ is to use naive SVM ranking. For some input $\boldsymbol{x}$, prediction of a label can be computed as $\operatorname{sgn}\left(\boldsymbol{x}^{T} \cdot \boldsymbol{w}+b\right)$, where $b$ is a bias term. The larger value of $w_{i}$ is, the more presence of feature $i$ inclines the classifier to predict positive class; the smaller (negative) it gets, the bigger $i$ th feature contribution towards negative class. When $w_{i}$ is close to 0 , it has minor influence on the output meaning that feature $i$ is not imporant in this context. Therefore naive SVM ranking can be defined as below:

$$
r_{i}=\left|w_{i}\right|
$$

However, in presence of highly corelated features it may be beneficial to apply Recursive Feature Elimination (RFE) 3]. In this iterative approach one feature is neglected in each run after a new SVM is trained on a working set of features. Ranking obtained this way, as opposed to naive approach, does not undervalue groups of correlated features. Note that ranking $\boldsymbol{r}$ is not limited to the two 


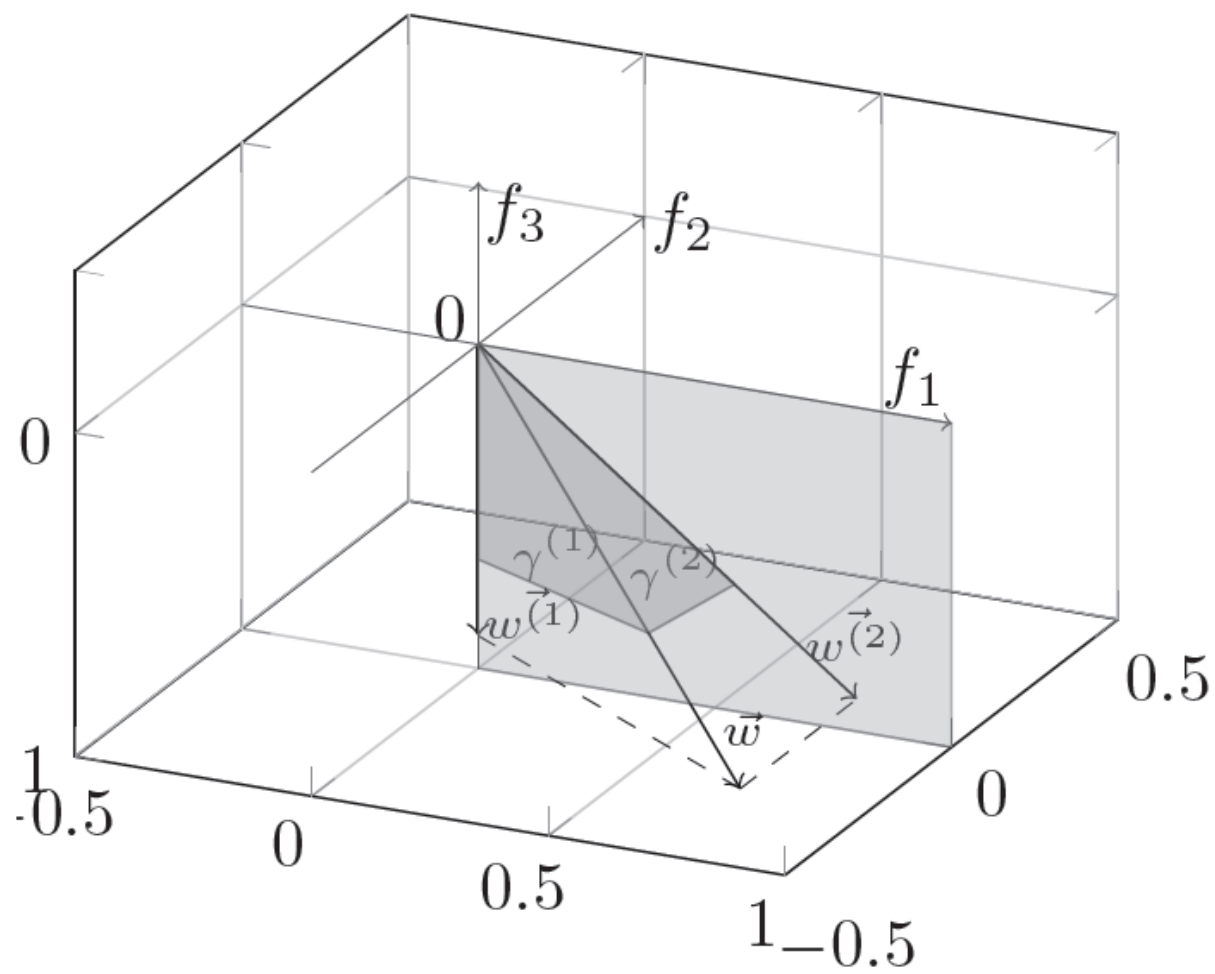

Fig. 1. Feature selection - toy example

approaches mentioned above. Features may be ranked in any way and exact rank values are not important since $r$ is only required to order features.

Figure 1 presents a toy feature selection scenario for a dataset of three features $f_{1}, f_{2}, f_{3}$. It was assumed that the trained hyperplane has normal vector $\boldsymbol{w}=[0.8 ;-0.35 ;-0.9]$ and naive SVM ranking was applied: $\boldsymbol{r}=[0.8 ; 0.35 ; 0.9]$ implying that features ordered by their ranks are $f_{3}, f_{1}, f_{2}$. In such case $\boldsymbol{w}^{(2)}=$ $[0.8 ; 0 ;-0.9]$ and $\boldsymbol{w}^{(2)}=[0 ; 0 ;-0.9]$. If $\alpha$ was set to $0.05, k=2$ features would be retained as $\cos \gamma^{(2)} \approx 0.96 \geq 1-\alpha$ and $\cos \gamma^{(1)} \approx 0.72<1-\alpha$.

\section{$3 \quad$ Experiments}

MATRIX'u application [9] was used to generate representations of three datasets originating from a Simple Wikipedia dump. Stemmed words were used as features and documents were expressed in vector space model with TF-IDF weighting. Features were then scaled to the range $[0.15,1]$. The datasets are described in Table 1. 
Table 1. Datasets used in the tests

\begin{tabular}{llccc}
\hline Name & Description & \# classes & \# objects \# features \\
\hline GR & categories Geography and Religion & 2 & 36000 & 90000 \\
TL & top level categories: Everyday Life, Ge- & 8 & 50000 & 115000 \\
& $\begin{array}{l}\text { ography, History, Knowledge, Litera- } \\
\text { ture, Media, People, Religion }\end{array}$ & & & \\
Science & subcategories of Science & 29 & 50000 & 117000 \\
\hline
\end{tabular}

Feature selection was performed with use of naive SVM ranking and various values of $\alpha$. Accuracy obtained after feature selection was compared to the result measured on all features. Figure 2 shows how the percentage of selected features depends on $\alpha$.

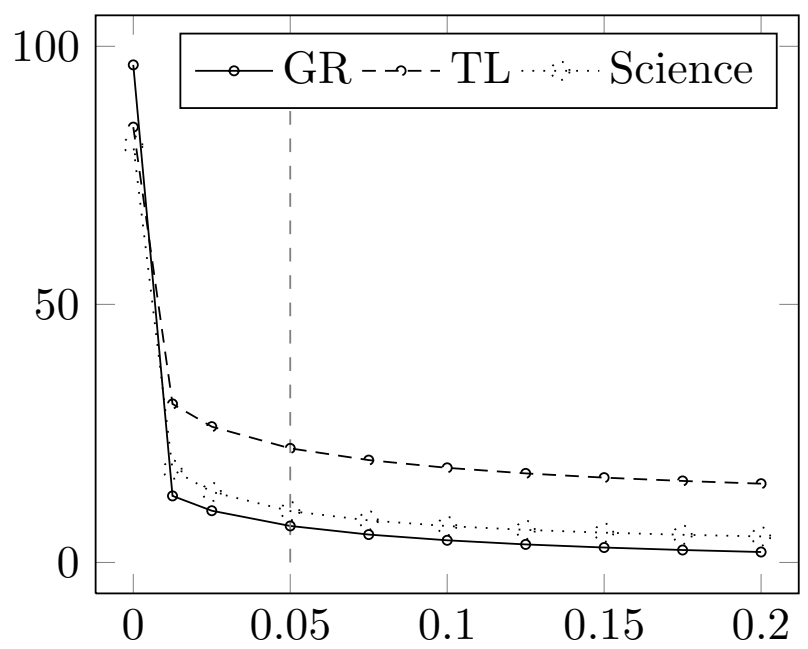

Fig. 2. Average percent of retained features (per hyperplane) as a function of the parameter $\alpha$

For each of the data sets there is a distinct elbow point: the number of features drops considerably for $\alpha=0.0125$ and stabilizes around $\alpha=0.05$. Percentages of retained features differ among the data sets. In Figure 3 the accuracy drop (measured performing 10-fold cross validation) is displayed. Note that relation between the relative accuracy and $\alpha$ is linear, and slopes of those lines differ depend on how many classes there are in a dataset. According to these results, the proposed approach to feature selection requires little parameter adjustment. For the considered data sets the only parameter of the described model universally yields good results for the steady value of $\alpha=0.05$.

A by-product of this feature selection method is the information that can be easily inferred from the vector $w$. Having ordered the features by $w_{i}$ one can find 


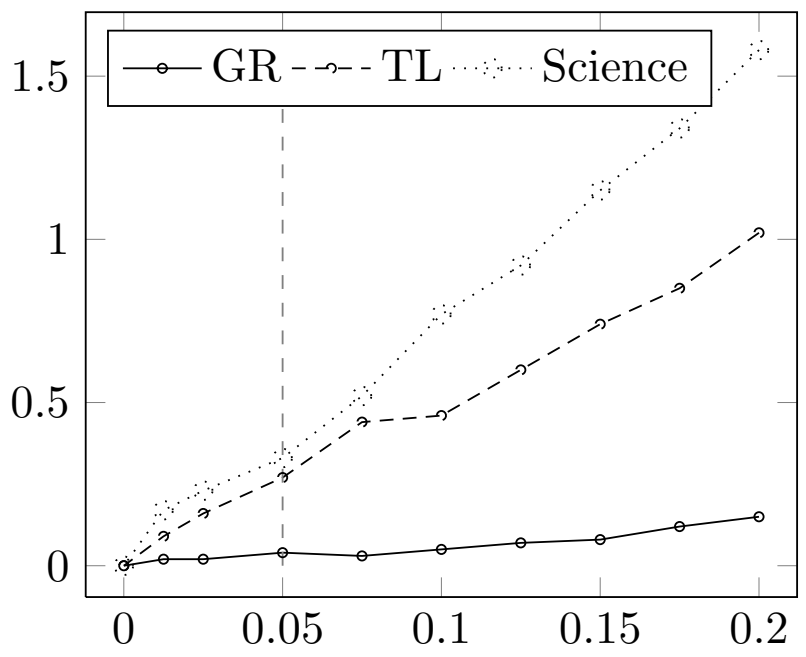

Fig. 3. Relative $F_{1}$-score accuracy drop as a function of $\alpha$ (percentage)

out what are the most descriptive features of a related class, which can be useful for debugging purposes. Table 2 presents keywords that are the most specific to some of the classes from the dataset TL. The words in the table are malformed due to stemming. Similarly, features most differentiating between two classes can be inspected this way - Table 3 presents words that are the most useful to tell Geography and Religion pages apart. Finally Table 4 lists features which are the most specific to category Mathematics as well as keywords indicating scientific categories other than math.

\section{Discussion}

We described a method for choosing the number of features that should be retained based on the hyperplane tilt angle caused by feature selection. This method is parametrized by a single parameter $\alpha$, however in our experiments on Wikipedia good results were achieved for constant value $\alpha=0.05$.

According to the conducted experiments, the method for selecting number of features allows for significant dimensionality reduction while preserving classification accuracy. Dimensionality of the datasets was decreased by from $78 \%$ to $90 \%$ while the relative accuracy drop was less than $0.5 \%$. With a single parameter value across various datasets containing different numbers of classes, we obtained satisfying dimensionality reduction. We plan to compare this technique to other known approaches in order to evaluate the order of features resulting from the ranking function [10].

The datasets differed in terms of "classification difficulty" (to distinguish between articles about geography and pages about religion is easier than to tell 
Table 2. Best describing keywords for some of the classes from the dataset TL

\begin{tabular}{ccccc}
\hline Geography & History & Literature & People & Religion \\
\hline town (2.9) & born (3.8) & book (5.8) & born (4.5) & religi (12.8) \\
river (2.7) & career (2.3) & fiction (4.9) & career (2.3) & mytholog (7.3) \\
city (2.6) & february (2.2) & screenwrit (4.1) & songwrit (2.2) & god (5.6) \\
island (2.2) & event (2.1) & treati (3.8) & ethnic (1.8) & cathedr (5.1) \\
county (2.0) & june (1.9) & newspap (3.8) & wrote (1.7) & church (4.5) \\
motto (1.9) & october (1.9) & diktat (3.0) & tribe (1.7) & anglican (4.3) \\
district (1.9) & military (1.7) & orig (3.0) & middleham (1.6) & mosqu (4.2) \\
chicago (1.9) & single (1.7) & epistl (3.0) & spous (1.5) & islam (4.2) \\
\hline
\end{tabular}

Table 3. Words differentiating between Geography and Religion

\begin{tabular}{cc}
\hline Geography & Religion \\
\hline citi (6.9) & religi (15.1) \\
town (4.2) & mytholog (6.5) \\
district (4.1) & god (6.2) \\
born (4.0) & christian (5.5) \\
armenian (3.8) & islam (5.2) \\
region (3.7) & cathedr (4.9) \\
area (3.7) & bibl (4.8) \\
river (3.7) & pope (4.2) \\
capit (3.7) & church (4.2) \\
franc (3.5) & jewish (3.9) \\
\hline
\end{tabular}

Table 4. Keywords specific to the category Mathematics along with the words indicating other subcategories of Science

\begin{tabular}{cc}
\hline \multicolumn{2}{c}{ Mathematics } \\
$+\quad$ & - \\
\hline mathemat (15.3) & fear (7.6) \\
number (7.1) & citi (6.9) \\
geometri (5.5) & comput (5.1) \\
math (5.3) & superscript (4.8) \\
algebra (4.7) & water (4.6) \\
actuari (4.6) & state (4.6) \\
arithmet (4.6) & town (4.6) \\
statist (4.4) & cyril (4.5) \\
function (4.2) & birth (4.2) \\
\hline
\end{tabular}

some scientific topics apart). Feature selection resulted in various numbers of retained features as well as various cut off thresholds. Such results would not be possible if the final dimensionality was fixed a priori or if only features ranked above some constant threshold were retained. 
Of course it would be easy to construct datasets for which optimal $\alpha$ would be vastly different. However, for groups of similar problems - in our case all problems origined in some areas of Wikipedia - good results can be obtained using constant value of $\alpha$.

Acknowledgments. The work has been supported by the Polish Ministry of Science and Higher Education under research grant N N 516432338.

\section{References}

1. Yang, Y., Pedersen, J.O.: A comparative study on feature selection in text categorization. In: Proceedings of the Fourteenth International Conference on Machine Learning, ICML 1997, pp. 412-420. Morgan Kaufmann Publishers Inc., San Francisco (1997)

2. Chen, J., Huang, H., Tian, S., Qu, Y.: Feature selection for text classification with naïve bayes. Expert Systems with Applications 36(3), 5432-5435 (2009)

3. Guyon, I., Weston, J., Barnhill, S., Vapnik, V.: Gene selection for cancer classification using support vector machines. Mach. Learn. 46(1-3), 389-422 (2002)

4. Brank, J., Grobelnik, M.: Feature selection using linear support vector machines (2002)

5. Hearst, M.A., Dumais, S.T., Osman, E., Platt, J., Scholkopf, B.: Support vector machines. IEEE Intelligent Systems and their Applications 13(4), 18-28 (1998)

6. Neumann, J., Schnörr, C., Steidl, G.: Combined svm-based feature selection and classification. Machine Learning 61(1-3), 129-150 (2005)

7. Rzeniewicz, J.: Analysis methods for intercategorial links. Master's thesis, Gdansk University of Technology (2013)

8. Fan, R.-E., Chang, K.-W., Hsieh, C.-J., Wang, X.-R., Lin, C.-J.: Liblinear: A library for large linear classification. J. Mach. Learn. Res. 9, 1871-1874 (2008)

9. Szymański, J.: Wikipedia Articles Representation with Matrix'u. In: Hota, C., Srimani, P.K. (eds.) ICDCIT 2013. LNCS, vol. 7753, pp. 500-510. Springer, Heidelberg (2013)

10. Balicki, J., Krawczyk, H., Rymko, Ł., Szymański, J.: Selection of Relevant Features for Text Classification with K-NN. In: Rutkowski, L., Korytkowski, M., Scherer, R., Tadeusiewicz, R., Zadeh, L.A., Zurada, J.M. (eds.) ICAISC 2013, Part II. LNCS (LNAI), vol. 7895, pp. 477-488. Springer, Heidelberg (2013) 